spine is the worst long-term outcome of this disease, although this occurs less frequently nowadays if compared to former decades. This fact might be related either to the natural course of the disease that becomes milder over time or to a consequent anti-inflammatory treatment initiated earlier in patients with axSpA.

Structural damage in the spine in axSpA is usually assessed on plain radiographs of the spine and, therefore, is frequently referred to as radiographic spinal progression. In the presentation, pathophysiology, assessment and ways of prevention and/or retardation of structural damage progression in the spine in patients with axSpA will be discussed.

Disclosure of Interests: Denis Poddubnyy Grant/research support from: AbbVie, Merck Sharp \& Dohme, Novartis, Consultant for: AbbVie, Bristol-Myers Squibb, Janssen, Merck Sharp \& Dohme, Novartis, Pfizer, UCB Pharma, Speakers bureau: AbbVie, Bristol-Myers Squibb, Janssen, Merck Sharp \& Dohme, Novartis, Pfizer, Roche, UCB Pharma

DOI: 10.1136/annrheumdis-2019-eular.8561

\section{SP0081 STRUCTURAL DAMAGE PROGRESSION IN PSA}

Philip Helliwell. University of Leeds, Leeds Institute of Rheumatic and Musculoskeletal Medicine, Leeds, United Kingdom

Psoriatic arthritis is a multifaceted disease in which the arthritis tends to be less frequent than in rheumatoid arthritis. This provides a challenge to measure radiographic progression using the simple and time-honoured fashion of assessing the hands and feet. Further, radiographic progression is slower in PsA and changes over a short time period, such as the placebo controlled phase of a trial, are minimal. Newer imaging, such as ultrasound and MRI may be more sensitive and responsive but data on these techniques are limited. These challenges, and how to overcome them, will be discussed.

Disclosure of Interests: Philip Helliwell Grant/research support from: Paid to charity: from AbbVie, Janssen and Novartis, Consultant for: Paid to charity: from AbbVie, Amgen, Pfizer, and UCB and Celgene. Paid to self: from Celgene and Galapagos

DOI: 10.1136/annrheumdis-2019-eular.8495

THURSDAY, 13 JUNE 2019

15:30:00 - 17:00:00

\section{Difficult to manage Sjögren's syndrome and Myositis}

\section{SP0082 SCREENING FOR MALIGNANCIES IN SJOGREN SYNDROME AND MYOSITIS}

Albert Selva-O'Callaghan. Vall d'Hebron General Hospital. Universitat Autonoma de Barcelona, Internal Medicine Dept, Barcelona, Spain

Both myositis and Sjögren syndrome may be associated with cancer. However, there are differences between the cancer-associated myositis and cancer that appears in patients with Sjogren syndrome, and these differences make for a distinct tumor screening approach. The fact that any type of cancer -mainly of the adenocarcinoma type- can be associated with myositis -dermatomyositis, either classical or amyopathic, immune-mediated necrotizing myopathy, or polymyositis phenotypes- makes more difficult to know if an occult malignancy is present. Nevertheless, in recent years biological markers such as several autoantibodies, essentially anti-TIF1 $\gamma$, but also anti-NXP2 and to some extent anti-HMGCR, and combined techniques such as PET/CT that allow to detect structural and functional changes by means of ${ }^{18} \mathrm{~F}$ - Fluorodeoxyglucose and whole CT have helped the clinician to screen for an occult cancer in patients with myositis. Alternatively, the clinical situation and screening approaches in Sjögren syndrome are more focused in the detection of lymphoproliferative disorders, mostly in salivary glands (MALT type lymphoma) but also marginal zone or diffuse large B cell lymphomas. Several approaches for detecting MALT-type lymphoma or generalized lymphomas, beyond the well-known parameters such as low complement levels, cytopenias, cryoglobulins or persistent enlargement of salivary glands, are also discussed.

\section{REFERENCES:}

[1] Selva-O'Callaghan A, Martínez-Gómez X, Trallero-Araguás E, Pinal-Fernández I. The diagnostic work-up of cancer-associated myositis. Curr Opin Rheumatol. 2018; 30:630-6

[2] Baldini C, Ferro F, Luciano N, Bombardieri S, Grossi E. Artificial neural networks help to identify disease subsets and to predict lymphoma in primary Sjögren syndrome. Clin Exp Rheumatol. 2018;36 (Suppl. 112): S137S144
Disclosure of Interests: None declared

DOI: 10.1136/annrheumdis-2019-eular.8586

THURSDAY, 13 JUNE 2019

15:30:00 - 17:00:00

\section{Anergy, exhaustion or post-activation in autoimmunity; Facts and future consequences}

\section{SP0083 MOLECULAR AND METABOLIC EVENTS WHICH UNDERWRITE T CELL PHENOTYPES IN AUTOIMMUNITY}

George Tsokos. Beth Israel Deaconess Medical Center/Harvard Medical School, Medicine, Boston, United States of America

The distribution and function of $\mathrm{T}$ cell subsets in patients with systemic lupus erythematosus (SLE) is aberrant. Distinct molecular and metabolic event dictate the numbers and function of $T$ cells. Specifically, the transcriptional repressor cyclic AMP response element modulator alpha (CREM $\alpha$ ), which is increased in cells from patients with SLE, accounts for the decreased expression of interleukin (IL)-2 and the increased expression of IL-17 through distinct epigenetic processes. CREM $\alpha$ promotes Th17 cell expansion by promoting the expression of Gls1, the first enzyme in glutaminolysis and by suppressing the expression of pyruvate dehydrogenase phosphatase catalytic subunit 2 that enables entry of pyruvate in to the Krebs cycle. In parallel, calcium calmodulin kinase 4 which is responsible for the increased binding of CREM $\alpha$ to cAMP response elements of the $I L-2$ and $I L-17$ loci, promotes the activity of pyruvate kinase $\mathrm{M} 2$ and promotes glycolysis and $\mathrm{TH} 17$ generation while suppressing the numbers of regulatory $\mathrm{T}$ cells. Understanding the exact molecular and metabolic processes that control $\mathrm{T}$ cell function in SLE enables therapeu tic considerations.

Disclosure of Interests: George Tsokos Grant/research support from: Janssen Research \& Development, LLC

DOI: 10.1136/annrheumdis-2019-eular.8604

\section{SP0084 POST-ACTIVATED B CELLS IN AUTOIMMUNITY}

Andreia Lino. Rheumatology and Clinical Immunology, Department of Medicine, Charité Universitätsmedizin Berlin and German Rheumatism Research Center Berlin (DRFZ), Berlin, Germany

Background: B cells are key players in autoimmune diseases such as systemic lupus erythematosus (SLE), primary Sjögren's syndrome (pSS) and rheumatoid arthritis (RA). In the past, it was believed that $B$ cells from these patients were hyperresponsive to $B$ cell receptor (BCR) and Toll-like receptor (TLR) signaling New insights suggest that $B$ cells from these patients present a post-activated functiotype.

Objectives: We aim at characterizing $B$ cells and B cell responses of SLE, pSS and RA patients to understand which venues can be taken towards new therapies for these diseases.

Methods: B cells and B cell subsets from peripheral blood samples from SLE, pSS and RA patients and healthy donors (HD), as well as few autoimmune tissues, were analyzed for: phosphotyrosine kinase (PTK) phosphorylation kinetics protein phosphatase activity, expression of phosphatases or of checkpoint molecules, such as e.g.PD-1, before and after BCR engagement, alone or together with TLR9 and CD40 stimulation, differentiation and proliferation. Expression of transcription factors, such as e.g.STAT1, and the effect of interferons in their expression in $\mathrm{B}$ cells from patients were also evaluated.

Results: B cell responses upon BCR engagement in SLE, pSS, RA patients were abnormal with diminished BCR downstream PTK phosphorylation. TLR9, but not CD40 responses were also abnormal. Part of the abnormality related to diffuse up-regulation of phosphatases that apparently counteracted PTK signaling. The abnormality was partially mimicked by repeated signaling through the BCR of HD $B$ cells and could be overcome by CD40 engagement. Check-point molecules, such as e.g.PD-1, was differentially expressed in SLE naive and memory B cells SLE naive and memory B cells expressed higher amounts of STAT1 compared to those of pSS, RA and HD.

Conclusion: Post-activated B cells are characterized by a phenotype of dysregu lated expression of certain check-point molecules, such as PD-1, some transcription factors, like STAT1 and certain phosphatases (figure 1). Our data suggest that CD40 activation is involved in modulating BCR responses in post-activated $B$ cells. This has implications for innovative therapies since blocking BCR signaling pathways and CD40 activation as well as targeting certain phosphatases may have synergistic value for treating systemic autoimmunity. 


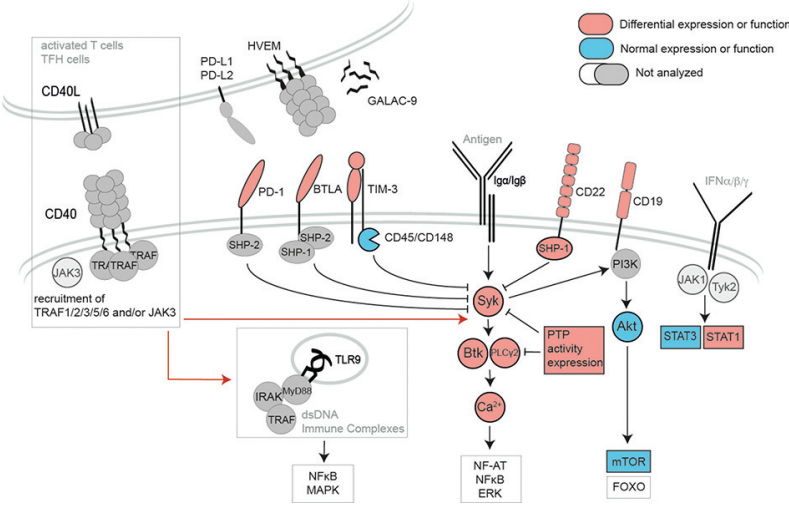

Abstract SP0084 Figure 1. Functiotypic characteristics of post-activated B cells from autoimmune patients.

Disclosure of Interests: None declared

DOI: 10.1136/annrheumdis-2019-eular.8527

THURSDAY, 13 JUNE 2019

15:30:00 - 17:00:00

Fracture liaison service: an opportunity for rheumatologists to focus on secondary fracture prevention

\section{SP0085 WHOM TO IDENTIFY AND INVITE TO AN FLS? WHO IS THAT FRACTURE PATIENT?}

\section{Bo Abrahamsen. $x, x, x$, Denmark}

Background: Though osteoporosis can be diagnosed through the presence of fragility fractures in the absence of other aetiology such as myeloma or metastases, patients with osteoporosis are also at increased risk of high impact traumatic fractures. Further, distinguishing between fracture mechanisms through chart review is often difficult and may lead to patients with osteoporosis being missed. On the other hand, focusing on identifying and treating patients at high imminent fracture risk rather than milder degrees of osteoporosis makes for better cost utility.

Objectives: To review the demographics of patients presenting with fragility fractures and the evidence for targeting osteoporosis assessment for each category of fracture in order to balance the need for maximum risk reduction with the requirement for an evidence based strategy.

Methods: Narrative review of recent epidemiology studies and national and international guidelines

Results: Based on epidemiology data from Iceland(1) and Denmark(2,3), the risk of subsequent fractures following a sentinel fracture event is critically dependent on the recency and the site of the initial fracture, with the risk of new fractures being particular high after major osteoporotic fractures(1) and pelvic fractures but less so after lower leg fractures(2).

In Danish women(2), 29.5\% of patients suffering a pelvic fracture went on to sustain a hip fracture in the next ten years, compared with $25.9 \%$ after a vertebral fracture but only $12.5 \%$ after a lower leg fracture. Further, despite the high recurrent fracture risk in the FLS setting, it is important to appreciate that the majority of hip fracture patients have not consulted with a prior fracture in the last ten years prior to their hip fracture(3).

The following issues will be addressed in more detail

1) Demographics of fracture patients with particular emphasis on age, sex and BMD.

2) Which fracture types are indicators of elevated risk of subsequent major osteoporotic fractures?

3) Which fracture types will respond to osteoporosis treatment?

4) What is the role of DXA in FLS?

5) Identifying vertebral fractures

Conclusion: FLS patients are at elevated risk of sustaining additional fractures both in the long term and in the short term, with risks being particularly high in the first years after the sentinel fracture and especially if the initial fracture is a pelvic fracture or a major osteoporotic fracture.

\section{REFERENCES:}

[1] Kanis, Osteoporos Int 2018, 29:1747-1757
[2] Hansen, Osteoporos Int 2015, 26:513-519

[3] Frederiksen, Osteoporos Int 2018; 29:109-114

Disclosure of Interests: None declared DOI: 10.1136/annrheumdis-2019-eular.8411

\section{SP0086 ASSESSMENT: HOW TO PERSONALIZE SECONDARY FRACTURE PREVENTION?}

Daniel Prieto-Alhambra. University of Oxford, NDORMS, Oxford, United Kingdom

Background: The existing armamentarium of therapeutic options to minimise fracture risk is growing. Different therapies have different properties, and can be used for different patient targets.

Objectives: To discuss data on short-term fracture risk following an index fracture, and on the short-term efficacy of different therapies.

Methods: Narrative review of recent literature.

Disclosure of Interests: Daniel Prieto-Alhambra Grant/research support from: Grants from Amgen, UCB Biopharma and Servier outside the submitted work, Consultant for: UCB Biopharma, Speakers bureau: Amgen DOI: 10.1136/annrheumdis-2019-eular.8564

\section{SP0087 TREATMENT: HOW TO IMPLEMENT IT AND HOW TO MONITOR ADHERENCE?}

Kassim Javaid Muhammad. University of Oxford, Nuffield Department of Orthopaedics, Rheumatology and Musculoskeletal Sciences, oxford, United Kingdom

Background: The key to achieving the benefits of an FLS on the population and the patient level is to ensure enough high-risk patients are initiated and then adhere to therapy. There is a low rate of primary and secondary adherence by patients with osteoporosis. Treatment initiation requires co-developed decision making with patients and clinicians balancing locally agreed intervention thresholds with effective and efficient risk and treatment communication. Treatment adherence depends on patient, treatment and healthcare service characteristics. Objectives: Describe the current status of treatment recommendation in the UK Describe the current methods for imrpoving adherence globally

Methods: Review of the data from the UK Fracture Liaison Service Database and IOF Capture the Fracture audits.

Disclosure of Interests: Kassim Javaid Muhammad Shareholder of: Zebra Medical Vision, Grant/research support from: Amgen, Kyowa Kirin Hakin, Consultant for: Amgen, Internis, Consilient Health,Mereo Biopharma, Kyowa Kirin Hakin, UCB, Speakers bureau: Amgen, Lilly UK, Internis, UCB

DOI: 10.1136/annrheumdis-2019-eular.8508

\section{THURSDAY, 13 JUNE 2019}

15:30:00 - 17:00:00

\section{How not to smoke like a chimney}

\section{SP0088 HOW YOU KNOW IT IS SMART NOT TO SMOKE LIKE A CHIMNEY}

Antonio Naranjo. Hospital Universitario de Gran Canaria Dr Negrin, Rheumatology, Las Palmas de Gran Canaria, Spain

Smoking is associated with a higher prevalence of many diseases. When a health professional is well informed of the risks of smoking in rheumatic diseases, the advice given to the patient about quitting smoking could be more effective.

It is known that smokers in the general population have an increased frequency of elevated rheumatoid factor (RF). Since 1987, we have known that cigarette smok ing is a risk factor for rheumatoid arthritis (RA), especially in men with RF-positive who are heavy smokers. Later, it could be verified that cigarette smoking is implicated in the pathogenesis of RA particularly when associated with antibodies, cyclic citrullinated peptides (ACPA) and the shared epitope. Smoking increases the risk of both subsets of RA with a more pronounced influence on the risk of ACPA positive RA. The polyclonal immune response against modified antigens in patients with RA is not exclusively citrulline-specific, carbamylation of antigens like vimentin could be involved in the pathogenesis of the disease. Thus, smoking is a risk factor for breaking tolerance to multiple autoantigens in RA.

In RA patients, cigarette smoking correlated with an increased risk of joint erosions, extra-articular manifestations and cardiovascular disease, in addition to the established effects of longstanding disease activity on the risk of myocardial infarction and stroke. 